J. Härkönen, E. Tuovinen, P. Luukka, H.K. Nordlund, and E. Tuominen, Magnetic Czochralski silicon as detector material, Nuclear Instruments and Methods in Physics Research A 579 (2007) 648-652.

(C) 2007 Elsevier Science

Reprinted with permission from Elsevier. 


\title{
Magnetic Czochralski silicon as detector material
}

\author{
J. Härkönen*, E. Tuovinen, P. Luukka, H.K. Nordlund, E. Tuominen \\ Helsinki Institute of Physics, CERN/PH, 1211 Geneva, Switzerland
}

Available online 24 May 2007

\begin{abstract}
The Czochralski silicon (Cz-Si) has intrinsically high oxygen concentration. Therefore $\mathrm{Cz}-\mathrm{Si}$ is considered as a promising material for the tracking systems in future very high luminosity colliders. In this contribution a brief overview of the Czochralski crystal growth is given. The fabrication process issues of $\mathrm{Cz}-\mathrm{Si}$ are discussed and the formation of thermal donors is especially emphasized. $\mathrm{N}^{+} / \mathrm{p}^{-} / \mathrm{p}^{+}$and $\mathrm{p}^{+} / \mathrm{n}^{-} / \mathrm{n}^{+}$detectors have been processed on magnetic Czochralski (MCz-Si) wafers. We show measurement data of AC-coupled strip detectors and single pad detectors as well as experimental results of intentional TD doping. Data of spatial homogeneity of electrical properties, full depletion voltage and leakage current, is shown and $\mathrm{n}$ and $\mathrm{p}$-type devices are compared. Our results show that it is possible to manufacture high quality $\mathrm{n}^{+} / \mathrm{p}^{-} / \mathrm{p}^{+}$and $\mathrm{p}^{+} / \mathrm{n}^{-} / \mathrm{n}^{+}$particle detectors from high-resistivity Cz-Si.

(C) 2007 Elsevier B.V. All rights reserved.
\end{abstract}

PACS: $71.55 .-\mathrm{i}$

Keywords: Particle detectors; Radiation hardness; Magnetic Czochralski silicon

\section{Introduction}

Particle detectors made of high-resistivity silicon wafers are widely used in high-energy physics experiments. Segmented silicon detectors provide excellent spatial resolution while being cost-effective due to well-established manufacturing technology. Nevertheless, particle radiation causes irreversible crystallographic defects in silicon material deteriorating the detector performance. First, the donor doping concentration of silicon is compensated by dominantly acceptor type defects. This may cause space charge sign inversion (SCSI) in n-type silicon material, which is followed by increase of the detector full depletion voltage $V_{\mathrm{fd}}$. Second, the leakage current of the detector $\left(I_{\text {leak }}\right)$ increases linearly as a function of accumulated radiation fluence. Third, due to the trapping of charge carriers, overall charge collection efficiency (CCE) degrades [1]. The deterioration of the CCE due to the trapping of charge carriers will be the most severe obstacle for the use of silicon sensors in future very high luminosity colliders with extremely harsh radiation environment.

\footnotetext{
*Corresponding author. Tel.: +4122 7671534; fax: +41227673600.

E-mail address: jaakko.haerkoenen@cern.ch (J. Härkönen).
}

Possible upgrade scenario of the LHC to a luminosity of $10^{35} \mathrm{~cm}^{-2} \mathrm{~s}^{-1}$, corresponding to expected total fluences of fast hadrons above $10^{16} \mathrm{~cm}^{-2}$ and reduced bunch-crossing interval of about $10 \mathrm{~ns}$ [2].

The detectors used in particle tracking systems must be fully depleted at reasonably low operating voltages. A practical limit of the operating voltage is $500 \mathrm{~V}$. Detectors have traditionally been fabricated on n-type high resistivity wafers made by Float Zone (Fz-Si) crystal growth technique. The low oxygen concentration in $\mathrm{Fz}-\mathrm{Si}$ is a drawback since oxygen has experimentally been found to improve the radiation hardness of silicon detectors, as demonstrated by the R\&D work performed in the framework of CERN RD48 collaboration $[3,4]$.

Developments in the crystal growth technology of Czochralski silicon $(\mathrm{Cz}-\mathrm{Si})$ have enabled the production of $\mathrm{Cz}$-Si wafers with sufficiently high resistivity and with well-controlled, high concentration of oxygen. In addition to the high oxygen concentration, $\mathrm{Cz}-\mathrm{Si}$ is available commercially in large quantities and many commercial detector foundries are familiar with its processing, since $\mathrm{Cz}-\mathrm{Si}$ is a basic raw material of the microelectronics industry. These aspects are important when designing a future large scale tracker system. 


\section{Crystal growth and material properties}

In the Czochralski method, polycrystalline silicon fragments are melted inside a silica crucible. During the process, argon gas is continuously flushing the interior of the crucible and the surface of the silicon melt. Silicon single crystals are grown by slowly pulling a crystal seed up from the molten silicon, thus developing an ingot. Later, wafers are cut from the ingot.

Oxygen concentration is one of the most important parameters of silicon wafers. For example, oxygen precipitates bind unwanted metallic impurities present during the processing of silicon devices [5,6]. Furthermore, stress induced during high-temperature processing can lead to the formation of slip defects in the wafer. The presence of oxygen stabilizes the wafer and thus $\mathrm{Cz}-\mathrm{Si}$ wafers are less prone to slip defect than $\mathrm{Fz}-\mathrm{Si}$ wafers [7].

During the crystal growth the silica crucible gradually dissolves, releasing oxygen, carbon and other impurities, namely aluminum and boron, into the silicon melt. Most of the oxygen vaporizes from the melt as silicon monoxide ( $\mathrm{SiO}$ ) that is transported away by the protection gas, but the rest stays in the melt and can dissolve into the single crystal. The concentrations of interstitial oxygen and carbon impurities in the single crystal are typically $(1-10) \times 10^{17} \mathrm{~cm}^{-3}$ and $(1-5) \times$ $10^{16} \mathrm{~cm}^{-3}$, respectively. If the oxygen concentration in silicon exceeds $1 \times 10^{18} \mathrm{~cm}^{-3}$, oxygen starts to precipitate, i.e. form clusters.

The homogeneity of the material is strongly affected by the thermal gradients present in the melt crucible. The thermal gradients cause strong buoyancy-driven convection and surface-tension-driven flows at the free surfaces of the melt. These melt-convective flows are at least partially turbulent and they are largely responsible for the crystal imperfections and radially and microscopically nonuniform distributions of dopant and impurity atoms in the crystals [8]. In addition, the silicon melt is enriched by dopants and therefore the resistivity decreases as a function of the length of the ingot. For these reasons, the maximum resistivity of the standard Czochralski wafers is rather limited.

The magnetic Czochralski (MCz) method is in principle the same as the standard Czochralski crystal growth method, but the ingot is grown in a strong magnetic field, which is used to dampen the oscillations in the silicon melt. That is, the Lorentz force resulting from the applied magnetic field influences the flow and reduces the amplitude of the melt fluctuations [9,10]. In this method the concentration and the distribution of oxygen can be controlled better than in the standard $\mathrm{CZ}$ method, which is important for large-area detector applications. The method is today widely used in the semiconductor industry and therefore wafers grown with $\mathrm{MCz}$ method have become available also for the detector applications.

\section{Processing of $\mathrm{MCz}-\mathrm{Si}$ detectors}

Pad detectors and segmented AC-coupled microstrip detectors have been processed on $\mathrm{n}$ and p-type $\mathrm{MCz}-\mathrm{Si}$ substrates at the Microelectronics Center of Helsinki University of Technology [11,12]. The wafers are $100 \mathrm{~mm}$ diameter double-side-polished $300 \pm 2 \mu \mathrm{m}$-thick having $\langle 100\rangle$ crystal orientation. The nominal resistivity, measured by the four-point probe method, of the borondoped wafers is $1800 \Omega \mathrm{cm}$, and $900 \Omega \mathrm{cm}$ in phosphorousdoped wafers. The oxygen concentration of these wafers was measured by the Fourier Transformation Infrared (FTIR) spectroscopy. The measurements were done on a thick reference wafer at the Institute of Electronic Materials Technology (ITME), Warszawa, Poland. The following oxygen concentrations were measured: $4.95 \times 10^{17} \mathrm{~cm}^{-3}$ (center), $4.89 \times 10^{17} \mathrm{~cm}^{-3}$ (right), $4.93 \times$ $10^{17} \mathrm{~cm}^{-3}$ (left) and $4.93 \times 10^{17} \mathrm{~cm}^{-3}$ (bottom).

The process sequence is basically similar when processing $\mathrm{MCz}-\mathrm{Si}$ and standard $\mathrm{Fz}-\mathrm{Si}$ devices: ion implantation of the segmented side and the back plane of the wafers, implantation activation thermal treatment, processing of bias resistors and strip dielectric, contact metallization, aluminum sintering and passivation. The essential difference between $\mathrm{MCz}-\mathrm{Si}$ and $\mathrm{Fz}-\mathrm{Si}$ is, however, the oxygen concentration. Oxygen can form at temperatures between 400 and $600{ }^{\circ} \mathrm{C}$ complexes consisting of four or more oxygen atoms. These complexes are called thermal donors (TD) and they influence electrical properties of detectors. When processing $\mathrm{p}^{+} / \mathrm{n} / \mathrm{n}^{+}$pin-diode detectors on $\mathrm{n}$-type phosphorous-doped $\mathrm{Cz}-\mathrm{Si}$ substrates, the TD formation decreases the effective bulk resistivity and consequently increases $V_{\mathrm{fd}}$. On the other hand, it is possible to tailor the $V_{\text {fd }}$ of the $\mathrm{n}^{+} / \mathrm{p}^{-} / \mathrm{p}^{+}$device made of p-type MCz-Si wafer by deliberate introduction of TD. The ion implantations doses in our process were all $1 \times 10^{15} \mathrm{~cm}^{-2}$ while the implantation energies were 70 and $30 \mathrm{keV}$ for phosphorous and boron, respectively. The thermal dry oxidations in our process were done at $1100^{\circ} \mathrm{C}$. After the oxidations, the temperature was ramped down at the rate $4{ }^{\circ} \mathrm{C} / \mathrm{min}$. The pullout of the wafers took place at $700{ }^{\circ} \mathrm{C}$. Thus the wafers were cooled as fast as possible in order to avoid the temperature range $400-600^{\circ} \mathrm{C}$. All detectors were passivated by approximately $60 \mathrm{~nm}$ thick silicon nitride $\left(\mathrm{Si}_{3} \mathrm{~N}_{4}\right)$ film grown by Plasma Enhanced Chemical Vapor Deposition (PECVD) method. The deposition temperature of PECVD $\mathrm{Si}_{3} \mathrm{~N}_{4}$ was $300{ }^{\circ} \mathrm{C}$.

Figs. 1(a) and (b) show the results of the capacitancevoltage (CV) and leakage current measurement of two n-type MCz-Si strip detectors.

The bias resistors of detectors have been processed with sputtered tungsten nitride $\left(\mathrm{WN}_{x}\right)$. The reason to use $\mathrm{WN}_{x}$ instead of commonly adopted polysilicon resistor technology is twofold. First, $\mathrm{WN}_{x}$ process is very simple. The resistors do not need ion implantation and subsequent dopant activation in order to adjust resistance value. The resistance value is adjusted by controlling the film 


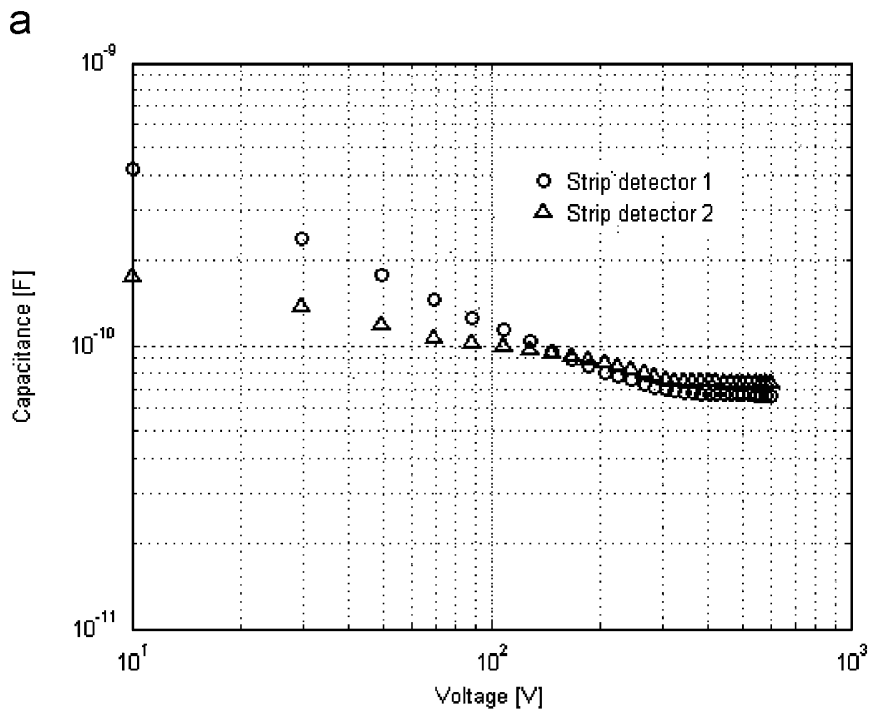

b

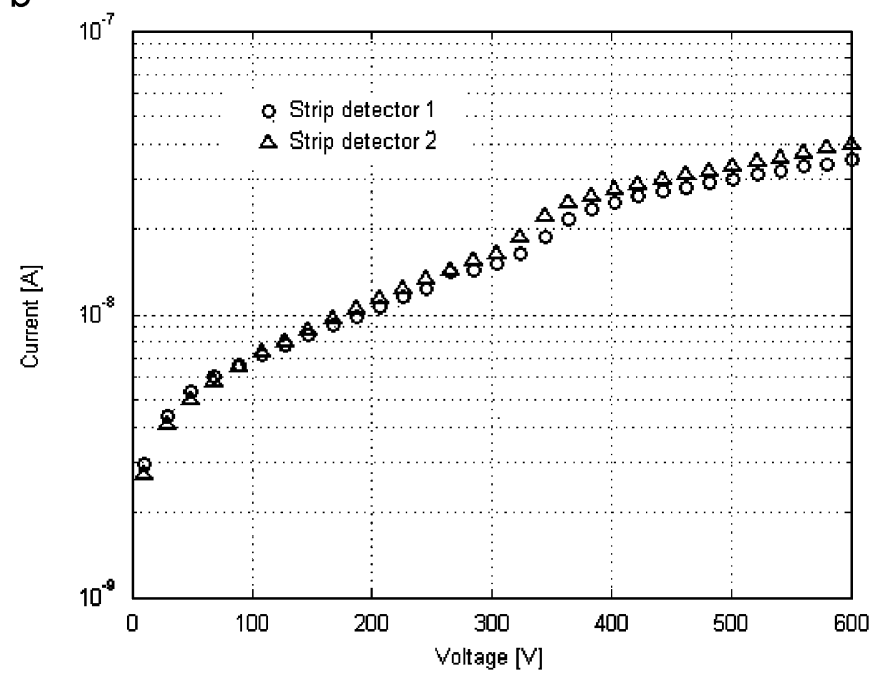

Fig. 1. (a) CV measurement of two mini strip detectors. The area of detectors is $2.5 \mathrm{~cm}^{2}$ and there are $1281.5 \mathrm{~cm}$ long AC-coupled strips. The $V_{\mathrm{fd}}$ of detectors are $307 \mathrm{~V}$ (1) and $304 \mathrm{~V}$ (2). (b) IV measurements of detectors (1) and (2). The leakage current at room temperature is about $16 \mathrm{nA}$ corresponding to about $6 \mathrm{nA} / \mathrm{cm}^{2}$.

thickness, which easily can be done with nanometer scale accuracy. Additionally, $\mathrm{WN}_{x}$ can be wet etched at room temperature completely selectively against underlying $\mathrm{SiO}_{2}$ by hydrogen peroxide [11]. Second, $\mathrm{WN}_{x}$ resistor is a room temperature process containing no hydrogen ingredients like silane $\left(\mathrm{Si}_{3} \mathrm{H}_{4}\right)$ used as a precursor in polysilicon process. This is important for the scope of controlling TD formation, which is known to be enhanced if hydrogen is present in the fabrication process at temperatures between 400 and $600{ }^{\circ} \mathrm{C}$. For the same reason, the strip insulators, $1 \mu \mathrm{m}$ thick stack of $\mathrm{SiO}_{2}$ and $\mathrm{Si}_{3} \mathrm{~N}_{4}$, have been deposited by PECVD at $300{ }^{\circ} \mathrm{C}$.

The process cleanliness has been monitored on a regular basis by minority carrier recombination lifetime measurements carried out by the microwave photoconductivity decay $(\mu \mathrm{PCD})$ method $[13,14]$. The measurements show no lifetime degradation in $\mathrm{MCZ}-\mathrm{Si}$ with respect to the standard $\mathrm{Fz}-\mathrm{Si}$ and experience the same process conditions and thermal history. Additionally, the results of recombination lifetime injection level dependence reported in Ref. [15] give reason to assume that the lifetime of $\mathrm{MCz}-\mathrm{Si}$ is not limited by the deep levels introduced, e.g., by copper or iron, but rather by relatively shallow levels.

\section{4. p-Type MCz-Si detectors}

The radiation hardness of silicon particle detectors can also be significantly increased by using $\mathrm{n}^{+} / \mathrm{p}^{-} / \mathrm{p}^{+}$structures instead of conventional $\mathrm{p}^{+} / \mathrm{n}^{-} / \mathrm{n}^{+}$detectors [16]. The essential advantage is that no SCSI occurs in p-type bulk, resulting in the collective junction to remain on the segmented side of the detector. Thus, the signal comes dominantly from electrons having three times higher mobility than that of holes and, consequently, the trapping of charge carriers within their trapping lifetime (inversely proportional to the irradiation fluence) is reduced. This allows higher charge collection in $\mathrm{n}^{+} / \mathrm{p}^{-} / \mathrm{p}^{+}$devices than in conventional detectors. If $\mathrm{p}$-type $\mathrm{Cz}-\mathrm{Si}$ is used as detector starting material, it is possible to engineer the full depletion voltage by compensating boron doping by TD.

In order to study TD generation, five $\mathrm{n}^{+} / \mathrm{p}^{-} / \mathrm{p}^{+} \mathrm{pad}$ detector wafers were sintered at $430{ }^{\circ} \mathrm{C}$ for different times, i.e. $35,45,60,70$ and $80 \mathrm{~min}$, at the peak temperature [12]. The thermal profile of sintering is pushin 2 min followed by the $10 \mathrm{~min}$ stabilization while the furnace settles again at the peak temperature (the furnace door is open during the pushin). After heating at the peak temperature the wafers were pulled out in $2 \mathrm{~min}$. In order to study the spatial uniformity of $V_{\mathrm{fd}}, 10$ diodes along each wafer diameter were picked for the $\mathrm{CV}$ measurements. The full depletion voltage of devices was extracted from the $\mathrm{CV}$ curve measured at room temperature and at $10 \mathrm{kHz}$ frequency. The effect of thermal treatment on effective doping concentration $N_{\text {eff }}$ is shown in Fig. 2.

An almost linear increase of $N_{\text {eff }}$ with respect to heating time can be seen in Fig. 2. The TD formation rate is discussed more in detail in Refs. [12,17,18].

The drawback of $n^{+} / \mathrm{p}^{-} / \mathrm{p}^{+}$devices is the more complex fabrication technology. Conventional detectors can be processed with 6-7 lithography mask levels while $\mathrm{n}^{+} / \mathrm{p}^{-} /$ $\mathrm{p}^{+}$devices require two more levels and additional ion implantations. The complexity is due to the positive dielectric charge that accumulates electrons near the surface inducing and inversion layer that short connects the segmented $\mathrm{n}^{+}$areas and draws very high leakage current from sawing damaged edges. Two methods are commonly adopted in order to suppress. P-spray is a light field implantation that results in the vicinity of the surface negative space charge that repulses electron accumulation. The so-called p-stop is simply heavily doped pn-junction isolation between segmented $\mathrm{n}^{+}$areas. Five $\mathrm{n}^{+} / \mathrm{p}^{-} / \mathrm{p}^{+}$pad detector wafers have been processed with different surface 


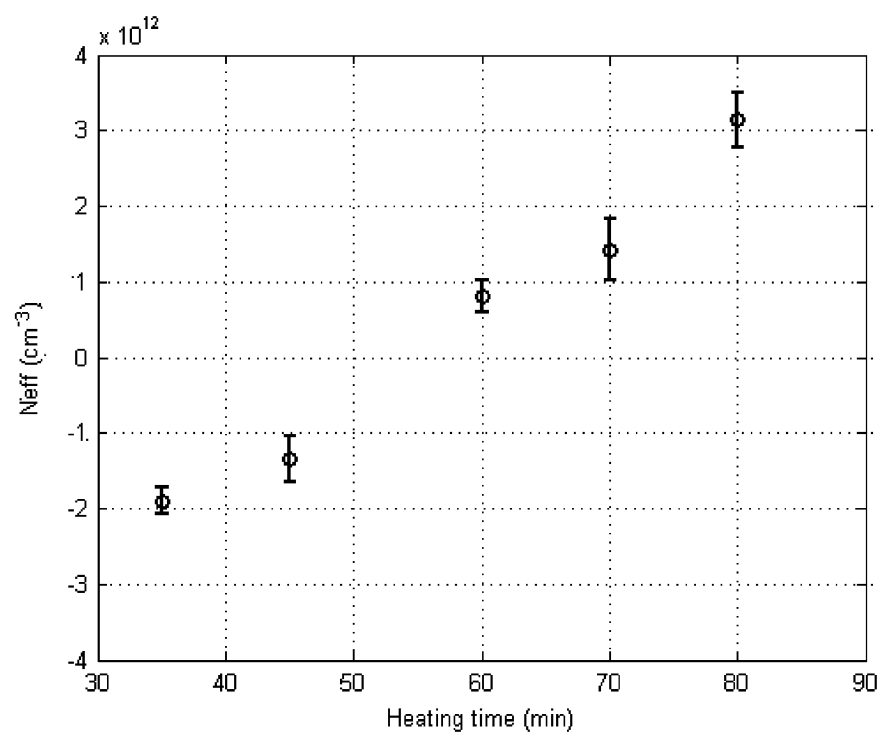

Fig. 2. Effect of thermal treatment on effective doping concentration.

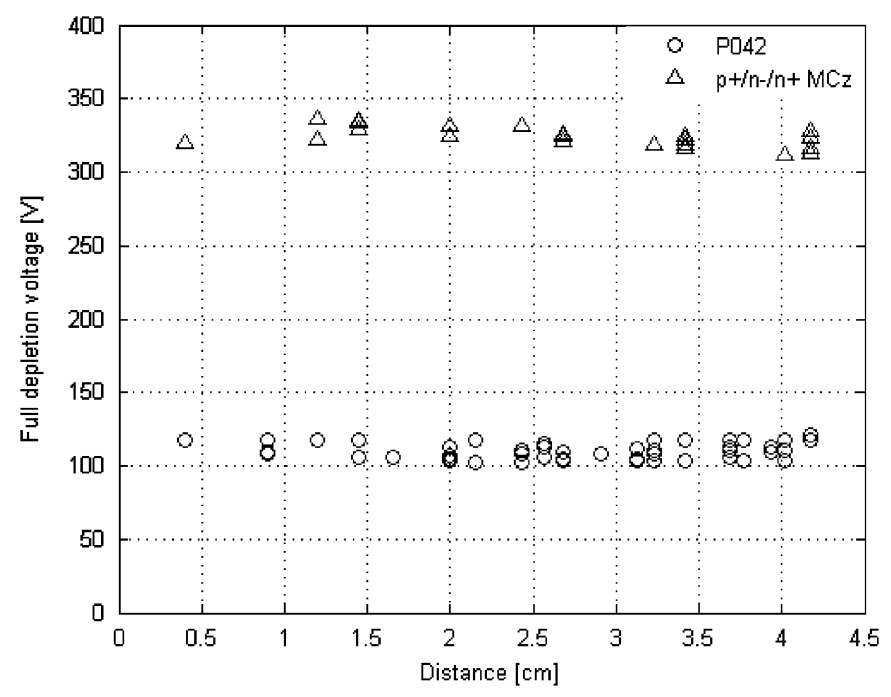

Fig. 3. $V_{\mathrm{fd}}$ as function of distance of p-type MCz-Si diodes $(O)$ and n-type MCz-Si diodes ( $(\mathbf{\Lambda})$.

terminations. The active pad implanted area of the diodes is $5 \times 5 \mathrm{~mm}^{2}$. It is surrounded by two wide guard rings $(100 \mu \mathrm{m})$ having opposite implantations (except wafer \#5) and 16 small guard rings (each $16 \mu \mathrm{m}$ wide) where every second guard ring has opposite doping. The distance between the active area implant and the first guard ring is $10 \mu \mathrm{m}$. The $V_{\mathrm{fd}}$ of as-processed diodes were measured by $\mathrm{CV}$. The $V_{\mathrm{fd}}$ of diodes originating from one p-type and one n-type wafer with respect to the distance from wafer center are shown in Fig. 3.

The properties of wafers are listed in Table 1.

The leakage current with respect to position in the wafer in $\mathrm{n}$ and p-type diodes are shown in Fig. 4.

It can be seen in Figs. 3 and 4 and Table 1 that the electrical properties of $\mathrm{n}^{+} / \mathrm{p}^{-} / \mathrm{p}^{+}$pad detectors are homogenous. The standard deviation of $V_{\mathrm{fd}}$ along the wafer
Table 1

P-spray and p-stop combinations of five pad detector wafer

\begin{tabular}{llllll}
\hline & $\begin{array}{l}\text { P-stop } \\
1 \times 10^{15} \mathrm{~cm}^{-2}\end{array}$ & P-spray & $\begin{array}{l}V_{\mathrm{fd}} \\
{[\mathrm{V}]}\end{array}$ & $\begin{array}{l}\mathrm{I}_{\text {leak }} \\
{[\mathrm{nA}]}\end{array}$ & $\begin{array}{l}\text { Yield } \\
{[\%]}\end{array}$ \\
\hline P042 & $\mathrm{X}$ & - & $110 \pm 5.5$ & $3.2 \pm 2.1$ & 93 \\
P068 & $\mathrm{X}$ & $1 \times 10^{12} \mathrm{~cm}^{-2}$ & $115 \pm 5.7$ & $2.6 \pm 2.0$ & 91 \\
P069 & $\mathrm{X}$ & $3 \times 10^{12} \mathrm{~cm}^{-2}$ & $115 \pm 4.4$ & $2.2 \pm 1.8$ & 84 \\
P082 & $\mathrm{X}$ & $5 \times 10^{12} \mathrm{~cm}^{-2}$ & $112 \pm 4.6$ & $3.3 \pm 2.4$ & 86 \\
P083 & - & $3 \times 10^{12} \mathrm{~cm}^{-2}$ & $106 \pm 4.4$ & $2.6 \pm 2.4$ & 58 \\
\hline
\end{tabular}

Each wafer consists of 80 diodes from which 57 were measured. The values of $V_{\mathrm{fd}}$ and $I_{\text {leak }}$ are expressed as average \pm standard deviation. The yield percentage refers to the number of bad samples out of 57 excluded from data. The sample is considered bad if breakdown occurs before the full depletion.

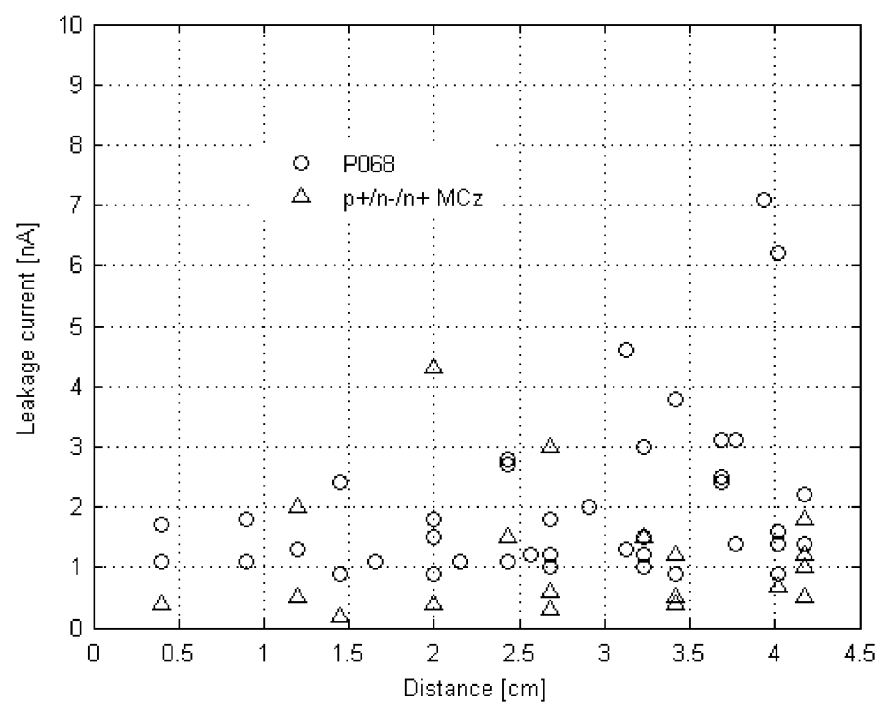

Fig. 4. Leakage current of $0.25 \mathrm{~cm}^{2}$ area diodes as function of distance from wafer center of p-type $\mathrm{MCz}-\mathrm{Si}$ diodes $(O)$ and n-type $\mathrm{MCz}-\mathrm{Si}$ diodes $(\mathbf{\Lambda})$

diameter is less than $5 \%$. The average $V_{\mathrm{fd}}$ value of five wafers undergo the same thermal history and processing condition for $106-115 \mathrm{~V}$. For comparison, in n-type devices the average $V_{\mathrm{fd}}$ is $324 \mathrm{~V}$ and the standard deviation along the wafer diameter is $7 \mathrm{~V}$. The leakage current is also satisfactory. The $I_{\text {leak }}$ is slightly higher in p-type diodes than in n-type references. That is obviously caused by surface current. A small trend of increasing $I_{\text {leak }}$ towards the wafer edges can be seen in Fig. 4. That is possibly due to the lower carrier lifetime near the wafer edges due to contaminants' diffusion from the wafer carriers during the high-temperature processing. The surface current isolation technology does not seem to influence $I_{\text {leak }}$ significantly. The breakdown properties are, however, the worst in P083 wafer having no p-stop and the best in P042 without any p-spray.

\section{Summary}

It is possible to manufacture high quality particle detectors from high resistivity $\mathrm{Cz}-\mathrm{Si}$. Electrical properties, 
i.e. full depletion voltage and leakage current, are comparable to those of traditional Float Zone silicon detectors. A characteristic feature of $\mathrm{Cz}-\mathrm{Si}$ is the formation of TD at certain temperatures. The kinetics of TD formation depends strongly on the oxygen concentration of $\mathrm{Cz}-\mathrm{Si}$ material, on the presence of hydrogen in semiconductor fabrication process and on the temperature. Therefore it is essential to control the thermal history and temperature homogeneity of the detector process. The use of magnetic field during the crystal growth might be necessary to adjust the oxygen concentration homogeneity to be sufficiently good for detector applications.

The $\mathrm{n}^{+} / \mathrm{p}^{-} / \mathrm{p}^{+}$and $\mathrm{p}^{+} / \mathrm{n}^{-} / \mathrm{n}^{+}$detectors show similar performance in terms of leakage current. However, if p-type $\mathrm{MCz}-\mathrm{Si}$ wafers are used to produce $\mathrm{n}^{+} / \mathrm{p}^{-} / \mathrm{p}^{+}$ devices, the TD formation can be utilized to tailor the $V_{\mathrm{fd}}$. The compensation of the acceptor doping does not increase the leakage current of the detector, as shown in Fig. 4. and Ref. [12]. This is expected because the TD form shallow levels in the band gap of silicon and thus their contribution to the leakage current is much smaller than, e.g., that of metallic impurities induced during the device processing. Therefore, detectors with high oxygen concentration and with low $V_{\mathrm{fd}}$ can be fabricated without additional process complexity or additional high temperature diffusion oxygenation.

Many investigations performed within the framework of CERN RD50 Collaboration have showed that MCZ-Si is less sensitive to the changes in $V_{\mathrm{fd}}$ in proton radiation, but no or very little improvement in neutron radiation. Also, no significant improvement in trapping lifetime and leakage current has been observed [19]. The lower $V_{\mathrm{fd}}$ under harsh radiation conditions is, however, itself an important improvement, because in the future large tracking systems upgrades, power dissipation will be one of the difficult engineering issues. Furthermore, $\mathrm{MCz}-\mathrm{Si}$ is available in large quantities and if necessary, in large diameter substrates. Thus, MCz-Si can be considered as a serious candidate for the detector material in future very high luminosity colliders.

\section{References}

[1] CERN-LHCC, RD50 status report 2002/2003, CERN-LHCC-2003058, 2003.

[2] F. Giannotti, et al., hep-ph/0204087, April 2002.

[3] G. Linstroem, et al. (RD48 Coll.), Nucl. Instr. and Meth. A 466 (2001) 308.

[4] A. Ruzin, et al., IEEE Trans. Nucl. Sci. NS-46 (5) (1999) 1310.

[5] B. Shen, et al., Appl. Phys. Lett. 70 (14) (1997) 1876.

[6] B. Shen, et al., J. Appl. Phys. 76 (8) (1994) 4540.

[7] W.M. Bullis, Mater. Sci. Eng. B72 (2000) 93.

[8] V. Savolainen, et al., J. Cryst. Growth 243 (2) (2002) 243.

[9] R.W. Series, et al., J. Cryst. Growth 113 (1991) 305.

[10] D.T.J. Hurle, et al., Phys. Lett. A43 (1973).

[11] J. Härkönen, et al., Nucl. Instr. and Meth. A 552 (2005) 43.

[12] M. Bruzzi, et al., Thermal donor generation in Czochralski silicon particle detectors, Nucl. Instr. and Meth., in press.

[13] J. Härkönen, et al., Nucl. Instr. and Meth. A 485 (2002) 159.

[14] D.K. Schroder, Semiconductor Material and Device Characterization, second ed., Wiley-Interscience, New York, 1998, pp. 429-437.

[15] J. Härkönen, et al., Mater. Sci. Semicond. Process. 9 (1-3) (2006) 261.

[16] G. Casse, Nucl. Instr. and Meth. A 487 (2002) 465.

[17] J. Härkönen, et al., Nucl. Instr. and Meth. A 552 (2005) 43.

[18] Y.J. Lee, et al., Phys. Rev. B 65 (2002) 085205.

[19] CERN-LHCC, RD50 status report 2005, CERN-LHCC-2005-037, 2005. 